\title{
Supernova Remnants and Star Formation in the Large Magellanic Cloud
}

\author{
Karna M. Desai ${ }^{1}$, You-Hua Chu ${ }^{1}$, Robert A. Gruendl ${ }^{1}$, William Dluger ${ }^{1}$, Marshall Katz ${ }^{1}$, Tony \\ Wong $^{1}$, C.-H. Rosie Chen ${ }^{2}$, Leslie W. Looney ${ }^{1}$, Annie Hughes ${ }^{3,4}$, Erik Muller ${ }^{5}$, Jürgen Ott ${ }^{6}$, Jorge
} L. Pineda ${ }^{7,8}$

\begin{abstract}
It has often been suggested that supernova remnants (SNRs) can trigger star formation. To investigate the relationship between SNRs and star formation, we have examined the known sample of 45 SNRs in the Large Magellanic Cloud to search for associated young stellar objects (YSOs) and molecular clouds. We find seven SNRs associated with both YSOs and molecular clouds, three SNRs associated with YSOs but not molecular clouds, and eight SNRs near molecular clouds but not associated with YSOs. Among the 10 SNRs associated with YSOs, the association between the YSOs and SNRs can be either rejected or cannot be convincingly established for eight cases. Only two SNRs have YSOs closely aligned along their rims; however, the time elapsed since the SNR began to interact with the YSOs' natal clouds is much shorter than the contraction timescales of the YSOs, and thus we do not see any evidence of SNR-triggered star formation in the LMC. The 15 SNRs that are near molecular clouds may trigger star formation in the future when the SNR shocks have slowed down to $<45 \mathrm{~km} \mathrm{~s}^{-1}$. We discuss how SNRs can alter the physical properties and abundances of YSOs.
\end{abstract}

Subject headings: Supernova Remnants; stars: formation; Magellanic Clouds

\footnotetext{
${ }^{1}$ Department of Astronomy, University of Illinois at Urbana-Champaign, 1002 West Green Street, Urbana, IL 61801, USA

${ }^{2}$ Department of Astronomy, University of Virginia, Charlottesville, VA 22904, USA

${ }^{3}$ Centre for Supercomputing and Astrophysics, Swinburne University of Technology, Hawthorn VIC 3122, Australia

${ }^{4}$ CSIRO Australia Telescope National Facility, P.O. Box 76, Epping NSW 1710, Australia

${ }^{5}$ Department of Physics and Astrophysics, Nagoya University, Chikusa-ku, Nagoya 464-8602, Japan

${ }^{6}$ National Radio Astronomy Observatory, P.O. Box O, Socorro, NM 87801, USA

${ }^{7}$ NASA Postdoctoral Program Fellow

${ }^{8}$ Jet Propulsion Laboratory, California Institute of Technology, 4800 Oak Grove Drive, Pasadena, CA 91109-8099, $U S A$
} 


\section{Introduction}

Star formation is frequently seen near supernova remnants (SNRs). For example, young stars have been detected in the vicinity of the Galactic SNR G54.1+0.3 (Koo et al. 2008), an SNR near the giant H II region N66 in the Small Magellanic Cloud (Gouliermis et al. 2008), and two SNRs in the Large Magellanic Cloud (LMC; Chu \& Gruendl 2008). Even the solar system appears to have formed at a site where a recent supernova explosion had occurred, as old meteorites show an anomalously high ${ }^{60} \mathrm{Ni}$ abundance that requires short-lived radioactive ${ }^{60} \mathrm{Fe}$ produced by core-collapse supernovae be injected into the nascent solar nebula (Vanhala \& Boss 2002; Mostefaoui et al. 2005; Tachibana et al. 2006).

The observed physical association between star formation and SNRs does not necessarily imply a causal relationship for two reasons. First, most massive stars are formed in clusters or OB associations where star formation may continue and propagate outward for a prolonged period of time; thus, SNRs produced by core-collapse supernovae are likely to be near young stars in star-forming environments. Second, theoretical calculations show that shocks at velocities of $20-45 \mathrm{~km} \mathrm{~s}^{-1}$ may compress molecular clouds to trigger star formation, while faster shocks will destroy molecular clouds (Vanhala \& Cameron 1998). SNRs are confirmed by their simultaneous exhibition of diffuse X-ray emission, nonthermal radio spectral index, and high [S II]/H $\alpha$ ratios (e.g., Mathewson et al. 1983), and these signatures are produced by high-velocity shocks. For example, X-ray-emitting plasma at temperatures $\geq 10^{6} \mathrm{~K}$ can be produced in adiabatic shocks only if the shock velocity is $\geq 300 \mathrm{~km} \mathrm{~s}^{-1}$. Therefore, confirmed SNRs have strong shocks that are destructive to molecular clouds. It has thus been suggested that the star formation observed near SNRs was triggered by the expansion of H II regions or wind-blown bubbles of the supernovae's massive progenitor stars (Koo et al. 2008; Gouliermis et al. 2008). This hypothesis is reasonable because small wind-blown bubbles with expansion velocities of $15-25 \mathrm{~km} \mathrm{~s}^{-1}$ are commonly observed in $\mathrm{H}$ II regions associated with young OB associations (Nazé et al. 2001) and large superbubbles formed by OB associations have typical expansion velocities of $20-45 \mathrm{~km} \mathrm{~s}^{-1}$ (Dunne et al. 2001).

We have used the sample of known SNRs in the LMC to make a broad investigation of the relationship between SNRs and star formation. The LMC sample of SNRs was initially compiled by Mathewson et al. (1983, 1984, 1985) using Einstein X-ray sources and radio observations. It has grown with additional members diagnosed by ROSAT X-ray observations (Chu et al. 1993, 1995, 1997; Smith et al. 1994) or by high [S II]/H $\alpha$ ratios (Points et al. 2004). The 45 currently known LMC SNRs are listed in Table 1. Recent star formation is best depicted by the presence of young stellar objects (YSOs). The LMC YSOs have been identified and inventoried by Gruendl \& Chu (2009, hereafter GC09) and Whitney et al. (2008) using Spitzer Space Telescope observations. As discussed in detail by GC09, the YSO catalog of Whitney et al. (2008) suffers problems of incompleteness and contamination by background galaxies, and therefore will not be used in this work. We have combined the LMC SNRs in Table 1 and the GC09 YSO catalog to search for star formation in juxtaposition with SNRs. We find 10 SNRs associated with YSOs, and 7 SNRs appear to be associated with molecular clouds but do not have YSOs. In this paper we describe the data 
sets and the search method in section 2, discuss star formation in the vicinity of SNRs in section 3 , and discuss the implications of this study in section 4 .

\section{Data Sets and Methodology}

To identify the location of SNRs in the LMC, we use $\mathrm{H} \alpha$, [O III], and [S II] images from the Magellanic Cloud Emission-Line Survey (MCELS; Smith \& The MCELS Team 1999). The MCELS images were taken with the Curtis Schmidt Telescope at the Cerro Tololo Inter-American Observatory (CTIO). The individual images have a pixel size of $2^{\prime \prime} .3$ pixel $^{-1}$, but the mosaicked images have a pixel size of $3^{\prime \prime}$. The [O III] filter includes only the $\lambda 5007$ line, while the $\mathrm{H} \alpha$ filter includes the $\mathrm{H} \alpha \lambda 6563$ and [N II] $\lambda \lambda 6548,6584$ lines, and the [S II] filter includes both the $\lambda 6716$ and $\lambda 6731$ lines. Thus, the MCELS H $\alpha$ images are really $\mathrm{H} \alpha+[\mathrm{N} \mathrm{II}]$ images, although the [N II] lines are usually less than $10 \%$ as strong as the $\mathrm{H} \alpha$ line because of the low nitrogen abundance of the LMC (Russell \& Dopita 1990). In general, the $\mathrm{H} \alpha$ images are best in showing both collisionally ionized gas in SNRs and photoionized gas in H II regions; the [O III] images show the cooling zone closest to SNR shock fronts and the gas photoionized by early-type O stars; and the [S II] images best delineate the boundaries of SNRs interacting with a dense medium. Young Type Ia SNRs in a mostly neutral medium are dominated by hydrogen Balmer lines, and hence visible only in the H $\alpha$ images (Chevalier et al. 1980).

We adopt the GC09 list of YSOs in the LMC. These YSOs, limited by the sensitivity and resolution of Spitzer observations, have masses greater than $\sim 4 M_{\odot}$ and correspond to intermediateand high-mass stars. To examine the location of YSOs relative to the SNRs, we have used archival Spitzer Space Telescope InfraRed Array Camera (IRAC; Fazio et al. 2004) images in the 3.6, 4.5, 5.8, and $8.0 \mu \mathrm{m}$ bands and Multiband Imaging Photometer for Spitzer (MIPS; Rieke et al. 2004) images in the 24 and $70 \mu \mathrm{m}$ bands, as used in GC09. The IRAC images have a resolution of $\sim 2^{\prime \prime}$, and the MIPS images have a resolution of $\sim 6^{\prime \prime}$ at $24 \mu \mathrm{m}$ and $\sim 17^{\prime \prime}$ at $70 \mu \mathrm{m}$. The IRAC $8.0 \mu \mathrm{m}$ images are useful in showing the polycyclic aromatic hydrocarbon (PAH) emission from partially ionized regions. They are also best for illustrating the locations of YSOs because of the combination of long wavelength and high angular resolution.

To examine molecular clouds associated with SNRs and star formation, we have used the CO $(\mathrm{J}=1 \rightarrow 0)$ observations from the NANTEN survey (Fukui et al. 2001, 2008) and Magellanic Mopra Assessment (MAGMA) survey (Ott et al. 2008; Hughes et al. 2010). The NANTEN observations were made with a 4 -m radio telescope with a 2 '.6 half-power beam and a velocity resolution of 0.65

$\mathrm{km} \mathrm{s}^{-1}$; this survey covered the entire LMC with a grid spacing of $2^{\prime}$. The $3 \sigma$ detection limit of the NANTEN survey corresponds to an integrated $\mathrm{CO}$ intensity of $I_{\mathrm{CO}}=1.2 \mathrm{~K} \mathrm{~km} \mathrm{~s}^{-1}$ or $N\left(\mathrm{H}_{2}\right) \sim 8 \times 10^{21} \mathrm{~cm}^{-2}$ using a conversion factor of $X_{\mathrm{CO}}=7 \times 10^{20} \mathrm{~cm}^{-2}\left(\mathrm{~K} \mathrm{~km} \mathrm{~s}^{-1}\right)^{-1}$. The NANTEN survey is estimated to be complete for molecular clouds with masses down to $5 \times 10^{4}$ $M_{\odot}$. All known SNRs in the LMC have been covered by the NANTEN survey. The MAGMA observations were made with the Mopra 22-m telescope with an angular resolution of $33^{\prime \prime}$ and a 
velocity resolution of $0.16 \mathrm{~km} \mathrm{~s}^{-1}$. The MAGMA survey covered only the $\sim 100$ brightest giant molecular clouds (GMCs) that had been previously detected by the NANTEN survey; thus, not every LMC SNR has MAGMA observations available. In this paper we have used integrated intensity maps from MAGMA smoothed to $45^{\prime \prime}$ resolution. An estimate of the MAGMA detection limit is $I_{\mathrm{CO}}=2 \mathrm{~K} \mathrm{~km} \mathrm{~s}^{-1}$ or $N\left(\mathrm{H}_{2}\right) \sim 1.4 \times 10^{22} \mathrm{~cm}^{-2}$ using the same conversion factor as used by Fukui et al. (2008). Since this is averaged over a $45^{\prime \prime}$ beam, most (if not all) clouds with emitting structures smaller than the NANTEN beam will be detected by MAGMA, but smoothly distributed emission that is just barely detected by NANTEN may escape detection in the MAGMA data. The range of cloud masses detected by MAGMA is $1.6 \times 10^{4}-6 \times 10^{6} M_{\odot}$ (Hughes et al. 2010). Small molecular clouds or low-density molecular gas associated with diffuse $8 \mu \mathrm{m}$ emission would not be detected by either survey.

The 45 LMC SNRs that we have examined are listed in Table 1. The coordinates and sizes of SNRs are measured from the MCELS images. Some SNRs are better defined in Chandra X-ray or Spitzer $24 \mu \mathrm{m}$ images, then these images are used to measure the SNRs' positions and sizes. We made a set of figures to identify the optical boundaries of the SNRs and compare them with the locations of YSOs and molecular clouds (a subset of these figures are presented in Figures 1 and 2). For each SNR, the figure contains three panels showing MCELS H $\alpha$, MCELS [S II], and Spitzer IRAC $8.0 \mu \mathrm{m}$ images with an identical field-of-view. Although SNRs are diagnosed by their higher $[\mathrm{S} \mathrm{II}] / \mathrm{H} \alpha$ ratios, the ratios are still $<1.0$ and most SNRs are still detected with the highest signalto-noise ratios in $\mathrm{H} \alpha$ images. YSOs are enshrouded by dust and thus appear as point sources in the $8.0 \mu \mathrm{m}$ images. The YSOs identified by GC09 are marked on the $\mathrm{H} \alpha$ and $8.0 \mu \mathrm{m}$ images. To show the distribution of molecular clouds, we have plotted the NANTEN and MAGMA CO contours over the $\mathrm{H} \alpha$ and $8.0 \mu \mathrm{m}$ images, respectively. The contour levels follow a geometric progression starting at the $3 \sigma$ level (i.e., $3 \sigma, 6 \sigma, 12 \sigma, 24 \sigma, \ldots$ ), where $\sigma$ is the typical root-mean-square noise level of the integrated intensity maps.

The positions of the YSOs have been marked with different colors and symbols depending on GC09's assessment of their nature. The YSOs marked in red have [8.0]<8.0 mag while those marked in green have [8.0]>8.0 mag, roughly corresponding to massive $\left(\gtrsim 10 M_{\odot}\right)$ and intermediate-mass $\left(\sim 4-10 M_{\odot}\right)$ stellar cores, respectively. The symbols used to mark the locations of the YSOs reflect the relative certainty that they are bona-fide YSOs. Open circles mark YSOs classified as "Definite YSOs" or "Probable YSOs," and crosses mark "Possible YSOs." The "Possible YSOs" are more likely to be non-YSOs than YSOs, but they can not be categorically ruled out that they are YSOs.

We have examined the figures carefully, comparing the relative locations of SNRs, YSOs, and molecular clouds. Since the LMC is a nearly face-on disk galaxy ( $\mathrm{i}=35^{\circ}$; van der Marel \& Cioni 2001), it is reasonable to assume association between two objects whose projected boundaries overlap. In our initial inspection, SNRs with YSOs projected within or in close vicinity are selected for further investigation. We use the NANTEN $3 \sigma$ contours for the boundary of molecular clouds and if the optical boundary of an SNR overlaps a molecular cloud we assume they are associated. This initial examination finds 3 SNRs associated with YSOs but not molecular clouds, 7 SNRs 
associated with both YSOs and molecular clouds, and 8 SNRs associated with molecular clouds but not YSOs, as listed in the last two columns in Table 1. The former 10 SNRs associated with YSOs are presented in Figure 1, and the latter 8 SNRs associated with molecular clouds in Figure 2. The associations between these SNRs and YSOs or molecular clouds are further evaluated using higher-resolution maps, such as MAGMA and ESO-SEST, and discussed in detail in the next section.

\section{Star Formation in the Vicinity of SNRs}

In this section we will first discuss the SNRs associated with YSOs, describing the relative locations of SNRs, YSOs, and molecular clouds. We further examine the relationships among YSOs, SNRs, and ionization fronts to probe possible triggering mechanisms for the star formation. Then we discuss the SNRs projected near molecular clouds but showing no star formation, and examine whether the SNRs are physically interacting with the molecular clouds.

\subsection{SNRs Associated with YSOs}

\section{SNR J0449.3-6920 (MCELS J0449-6921)}

This SNR has a diameter of $2^{\prime}(30 \mathrm{pc})$. The SNR shell, best seen in the [S II] image, is well defined on the north and south sides. The $\mathrm{H} \alpha$ image shows that the SNR blends with $\mathrm{H}$ II regions to its west and east. Two YSOs are present in the vicinity of the SNR. The $\mathrm{H} \alpha$ image shows that one YSO is located in a dark cloud to the east of the eastern $\mathrm{H}$ II region, and hence it is not associated with the SNR. The other YSO is located on the eastern rim of the western H II region. As the YSO is located in the partially ionized surface of the western diffuse H II region (the large elliptical shell structure in the $8.0 \mu \mathrm{m}$ image), it is most likely that its formation was triggered by the expansion of this western diffuse H II region. No molecular clouds in the vicinity of the SNR were detected by NANTEN.

\section{SNR J0455.6-6838 (N86)}

This SNR has a multiple-lobe structure, with the longest dimension approaching 100 pc (Williams et al. 1999). The northwest corner of the SNR borders the southern extension of the NANTEN GMC J0455-6830 (Fukui et al. 2008). The MAGMA observations covered the core of this GMC but not the southern extension. A YSO is detected near the border between the SNR and the GMC. As the exact boundary of the SNR is not known, it is not clear whether the YSO is inside the SNR boundary and physically associated, or outside the boundary and unrelated to the SNR.

SNR J0513.2-6912 (SNR 0513-692) 
This SNR is $4.5 \times 3.2(68 \mathrm{pc} \times 48 \mathrm{pc})$ in size. The northeastern quadrant of the SNR overlaps a small molecular cloud that was detected but not cataloged by NANTEN (Fukui et al. 2008). No MAGMA observations are available for this small cloud. In the region where the SNR overlaps the molecular cloud, there exist a small dense H II region and three YSOs. The $8.0 \mu \mathrm{m}$ image shows that the YSOs are located along filaments that extend to the northeast and belong to a larger structure unrelated to the SNR.

To show the detailed relationship among the small H II region, SNR, YSOs, and the $8.0 \mu \mathrm{m}$ filaments, we present in Figure 3 a close-up, high-resolution $\mathrm{H} \alpha$ image obtained with the MOSAIC camera on the CTIO 4m telescope (from GC09), along with the $8.0 \mu \mathrm{m}$ image. The H $\alpha$ image shows that the massive YSO is coincident with the brightest $\mathrm{H} \alpha$ knot, which is separated from the rest of the $\mathrm{H}$ II region by a dust lane. One intermediate-mass YSO is located in the southwest extension of the dust lane, while the other intermediate-mass YSO is located at 5 pc from the southern edge of the $\mathrm{H}$ II region.

The close alignment of the three YSOs along the SNR rim may lead to the impression that the SNR triggered the formation of these YSOs; however, this speculation is not supported by the dynamic timescale of the SNR and the ages of the YSOs. The SNR J0513.2-6912 has an average radius of $30 \mathrm{pc}$. Although its expansion velocity $\left(V_{\exp }\right)$ is unknown, we may adopt the typical values of 150-300 $\mathrm{km} \mathrm{s}^{-1}$ observed in confirmed SNRs in the Magellanic Clouds (Chu \& Kennicutt 1988). Assuming a Sedov phase for the SNR, its dynamic age would be $4-8 \times 10^{4} \mathrm{yr}$. The interaction of the SNR and the molecular cloud occurred only during the last $\sim 20 \%$ of its lifetime, i.e., $0.8-1.6 \times 10^{4}$ yr. This timescale is much shorter than the contraction timescale of intermediate-mass stars, $\sim 10^{6}$ yr, and massive stars, $\sim 10^{5}$ yr (Bernasconi \& Maeder 1996). Thus, it is impossible for the SNR to have triggered the formation of these YSOs.

One possible cause for the close alignment of YSOs and SNR rim is that the supernova exploded inside an interstellar bubble blown by its progenitor massive star. Interstellar bubbles have mild expansion velocities, $15-25 \mathrm{~km} \mathrm{~s}^{-1}$ (Nazé et al. 2001), and may trigger star formation. In this case, the supernova ejecta initially expands rapidly in the low-density interior of the bubble and slows down when it encounters the dense bubble shell; thus, the SNR boundary is dictated by the previous interstellar bubble and the YSOs triggered by the interstellar bubble naturally appear along the SNR shell rim.

\section{SNR J0532.5-6731 (SNR 0532-675 in N57)}

This SNR is in the northern portion of the superbubble in LHA120-N57 (Henize 1956). It was

first suggested by Mathewson et al. (1985), and is best shown in recent XMM-Newton observations (Maddox et al. 2010, in preparation). X-ray contours extracted from these X-ray observations are overplotted on the [S II] image to show the boundary of the SNR, 4.'5 in diameter. The NANTEN GMC J0532-6730 is detected on the northeast side of the SNR, but the MAGMA map shows no overlap between the GMC and the SNR. One YSO is detected within the GMC, but it is projected outside the boundary of the SNR, indicating that the SNR and its progenitor are not responsible 
for the formation of this YSO.

\section{SNR J0535.7-6602 (N63A)}

The SNR is expanding within a bubble produced by its progenitor within the N63 H II complex. X-ray contours extracted from Chandra observations (Warren et al. 2003) are overplotted on the [S II] image to show the boundary of the SNR, $\sim 1.2$ in diameter. No molecular clouds are detected by NANTEN. Two YSOs are projected within the boundary of N63, but well outside the SNR. It is likely that the expansion of the N63 H II region triggered the formation of these YSOs. This suggestion is further supported by the location of one YSO in the partially ionized eastern rim of the N63 H II region (see the $8.0 \mu \mathrm{m}$ image in Figure 1). A detailed study of star formation in the H II region N63 has been reported by Caulet et al. (2008).

\section{SNR J0536.0-6735 (DEM L241)}

This SNR has a shell like structure and is associated with the OB association LH88 (Lucke \& Hodge 1970); it has a relatively small expansion velocity, $\sim 70 \mathrm{~km} \mathrm{~s}^{-1}$, suggesting that the SNR may be expanding into a stellar-wind blown bubble (Chu 1997). The SNR overlaps the NANTEN GMC J0535-6735. This GMC is resolved by MAGMA observations into clumps, and the two largest clumps contain multiple YSOs. These molecular clumps and the YSOs are unrelated to the SNR. One YSO is projected within the SNR, but as this SNR is superposed on an OB association, it is uncertain whether this YSO is truly associated with this SNR and its progenitor or with other members of LH88.

\section{SNR J0537.5-6627 (DEM L256)}

This SNR has also been identified as MCELS J0537-6628. The northern part of this SNR overlaps the NANTEN GMC J0537-6626. One massive and two intermediate-mass YSOs are detected on the northwest rim of the SNR within the region overlapping the GMC. The $8.0 \mu \mathrm{m}$ image shows that the three YSOs are aligned along an arc of PAH emission. To provide a detailed view of this region, we present a high-resolution $\mathrm{H} \alpha$ close-up image, along with the $8 \mu \mathrm{m}$ image, in Figure 4. We have also analyzed the underlying stellar population using the Magellanic Cloud Photometric Survey (Zaritsky et al. 2004), and identified one star within the mass range 20-25 $M_{\odot}$ ( O 8 spectral type, marked with a blue triangle in Figure 4$)$ and two stars within $15-20 M_{\odot}(\sim \mathrm{B} 0$ spectral type, marked with orange triangles in Figure 4). The two less massive stars are inside small H II regions and one is coincident with an intermediate-mass YSO. While these YSOs and early-B stars may contribute to the excitation of PAHs along the $8 \mu \mathrm{m}$ arc, the surface brightness of the $8 \mu \mathrm{m}$ arc suggests nearly uniform illumination/excitation, which is more naturally provided by the $\mathrm{O} 8$ star at a distance (near the southeast corner of Figure 4).

High-dispersion echelle spectra of this SNR show a non-uniform expanding shell with the largest expansion velocity occurring on the receding side and reaching $\sim 120 \mathrm{~km} \mathrm{~s}^{-1}$ (Klimek et al. 2006). Adopting this as the expansion velocity and assuming a Sedov phase, the dynamic age of the SNR is $\sim 8 \times 10^{4} \mathrm{yr}$. If we further assume that the interaction of SNR and the YSOs' natal cloud 
occurred during the last $20 \%$ of the SNR lifetime, there would not be sufficient time for the clouds to contract and form these YSOs. Therefore, similar to SNR J0513.2-6912, SNR J0537.5-6627 cannot have triggered the formation of the YSOs along its northwest rim. Likewise, the supernova most likely exploded in the central cavity of a bubble blown by its massive star progenitor, and the YSOs triggered by the bubble expansion would naturally fall along the SNR rim.

\section{SNR J0537.8-6910 (N157B)}

This SNR is in an H II region ionized by the OB association LH99 (Chu 1997). With a complex nebular background, the SNR boundary is best determined using nebular kinematics (Chu et al. 1992) or diffuse X-ray emission (Chen et al. 2006). A low-level X-ray contour is plotted in Figure 1 to illustrate the rough boundary of this SNR. The NANTEN survey shows a large molecular complex over the SNR, but the molecular complex is resolved by MAGMA observations into clumps. No molecular peak is detected within the SNR boundary, although numerous dark clouds or dusty features are seen. Two massive and three intermediate-mass YSOs are detected within the boundary of the SNR, with the two massive ones deeply embedded in dark clouds. The complexity in this H II region precludes unambiguous associations between the YSOs and the SNR.

\section{SNR J0540.0-6944 (SNR in N159)}

This SNR is associated with N159 (Chu et al. 1997; Williams et al. 2000). N159 is an active star forming region that hosts the OB association LH105. The NANTEN data show that N159 is associated with the northern end of the large molecular ridge of the LMC. The MAGMA map has

resolved this region into multiple concentrations (Ott et al. 2008) and shows that the YSOs are projected near the molecular peaks. This region is too complex to make unambiguous association between YSOs and the SNR. A detailed discussion of the star formation in N159 has been reported by Chen et al. (2010).

\section{SNR J0543.1-6858 (DEM L299)}

This large SNR shows a central cavity of dimension $1 ! 8 \times 2 ! 3(27 \mathrm{pc} \times 35 \mathrm{pc})$. A YSO is projected within a small bubble-like structure, $\sim 1^{\prime}$ across, at the northwest rim of the SNR. The 8.0 $\mu \mathrm{m}$ image shows that the YSO is located in a ridge of enhanced diffuse emission, which is most likely $\mathrm{PAH}$ emission at the boundary between ionized and neutral media. A close-up, high-resolution $\mathrm{H} \alpha$ image of this small H II region is shown in Figure 5, along with an $8.0 \mu \mathrm{m}$ image. The curved ridge of $8.0 \mu \mathrm{m}$ emission is centered on a bright star that has been cataloged as Sk-68 155 and classified as a B0.5 (based on optical data) and O8 Ia (based on UV data) by Misselt et al. (1999). Using photometric measurements of Sk-68 155, $U=12.04, B=12.76$, and $V=12.72$ (Nicolet 1978), we estimate a spectral type of $\sim \mathrm{B} 0 \mathrm{Ia}$, roughly consistent with the previous classifications. With this spectral type, $\mathrm{Sk}-68155$ is able to photoionize this H II region, excite the PAHs, and possibly trigger the formation of the YSO. This small H II region and its associated PAH features and YSO are exterior to the SNR boundary, and thus not triggered by SNR J0543.1-6858. 


\subsection{Other SNRs Projected Near Molecular Clouds}

Eight SNRs are projected near molecular clouds, but no YSOs are observed within the SNR boundaries. Figure 2 shows images of these SNRs. Close examinations of the NANTEN and MAGMA contours in Figure 2 reveal that four SNRs do not seem to be in direct contact with molecular clouds: J0518.7-6939 in N120, J0521.6-6543, J0523.1-6753 in N44, and J0550.5-6823. In the case of J0526.0-6604 (N49), the SNR overlaps with a small feature in the MAGMA map, but the feature is smaller than the MAGMA resolution and the channel maps do not detect this feature in any adjacent velocity channels, suggesting that it is just a noise bump. A deep ESO-SEST CO $\mathrm{J}=2 \rightarrow 1$ observation shows a small cloud, with a mass of $9 \times 10^{3} M_{\odot}$ assuming $N_{\mathrm{H}_{2}} / W_{\mathrm{CO}(2-1)}=$ $1.87 \times 10^{21} \mathrm{~cm}^{-2}\left(\mathrm{~K} \mathrm{~km} \mathrm{~s}^{-1}\right)^{-1}$, on the eastern rim of N49 (Banas et al. 1997). This small cloud would be below the detection limit of both the NANTEN and MAGMA surveys. In the case of SNR N186D, the large beam size and unavailability of the channel maps of the NANTEN survey make it impossible to assess whether the SNR is truly interacting with the molecular cloud.

The remaining two SNRs, N103B and N132D, are projected within GMCs in both the NANTEN and MAGMA surveys. The SNR N103B has been shown to be the descendant of a Type Ia su-

pernova based on the abundances derived from X-ray observations (Hughes et al. 1995; van der Heyden et al. 2002). As the progenitor of the Type Ia supernova is not a massive star with ionizing power, there was no H II region or wind-blown bubble to trigger star formation in the GMC; therefore, the lack of YSOs around the SNR N103B is expected. In the case of N132D, high-resolution ESO-SEST observations ( $\mathrm{CO} \mathrm{J}=2 \rightarrow 1$; Banas et al. 1997) show that the molecular cloud is south of the SNR boundary. The apparent overlap between the molecular cloud and N132D in the NANTEN and MAGMA maps is caused by lower resolution and the smoothing of the data. Based on the highresolution ESO-SEST results, we do not see clear indication that the SNR or its progenitor has significantly interacted with the GMC; therefore, it is not surprising that no YSOs exist around the SNR N132D.

\section{Discussion and Conclusions}

The SNR-triggered star formation mechanism has been of great interest, but is frequently suggested casually without substantiation. In order to study the current and possible future star formation associated with SNRs, we have examined the entire sample of 45 SNRs in the LMC and assessed their relationship with YSOs and molecular clouds. Initial examinations using only the NANTEN maps of molecular clouds found seven SNRs associated with both YSOs and molecular clouds, three SNRs associated with YSOs but not molecular clouds, and eight SNRs near molecular clouds but not associated with YSOs. These 18 SNRs were further scrutinized with higher-resolution molecular maps in conjunction with Spitzer IRAC $8.0 \mu \mathrm{m}$ images.

Among the 10 SNRs associated with YSOs, two (N157B and the SNR in N159) are in regions with very active star formation, so it is difficult to assess their relationship with the YSOs. In 
four cases, either the YSOs are clearly outside the SNR boundary (N63A and the SNR in N57), or the association between YSOs and the SNRs is uncertain (N86 and DEM L241). In the remaining four cases, YSOs are projected near the SNR rims. Upon closer examination, the YSOs near J0449.3-6920 and DEM L299 are located within $8.0 \mu \mathrm{m}$ filaments associated with neighboring H II regions, and hence not triggered by the SNRs. Only SNRs J0513.2-6912 and DEML256 have YSOs closely aligned along their rims; however, the time elapsed since the SNR began to interact with the YSOs' natal clouds is much shorter than the contraction timescales of the YSOs, and thus the YSOs could not have been triggered by the SNRs.

From the sample of confirmed LMC SNRs, we do not see any evidence of SNR-triggered star formation. This result is consistent with that of theoretical considerations of SNR shocks interacting with molecular clouds: young SNRs with powerful shocks destroy molecular clouds, but older SNRs whose shock velocities have slowed down to $<45 \mathrm{~km} \mathrm{~s}^{-1}$ may compress molecular clouds to form stars (Vanhala \& Cameron 1998). However, observationally it is difficult to confirm or refute SNRtriggered star formation because such old SNRs have already lost their distinguishing characteristics at radio and X-ray wavelengths.

The frequent occurrence of star formation near young SNRs resulting from core-collapse supernovae should not be surprising because massive stars tend to form in clustered environment where expanding $\mathrm{H}$ II regions and interstellar bubbles can trigger further star formation. In our study of LMC SNRs, we find at least five SNRs (J0449.3-6920, J0513.2-6912, N63A, DEM L256, and DEM L299) having YSOs located along arcs or filaments of PAH emission in the $8 \mu$ m images, suggesting that the star formation was triggered by advancing ionization fronts or expanding H II regions.

Fifteen of the SNRs in the LMC are near molecular clouds, and in some cases the SNRs may have already interacted with the molecular clouds, but the fast SNR shocks cannot trigger star formation yet. As the SNRs age $\left(10^{5}-10^{6} \mathrm{yr}\right)$ and their shock velocities have slowed to $<45 \mathrm{~km} \mathrm{~s}^{-1}$, their interactions with the molecular clouds may trigger star formation. About $33 \%(15 / 45)$ of the known SNRs in the LMC have the potential to trigger star formation in the future. It is interesting to note that when SNRs can trigger star formation, the currently associated massive YSOs would have become more evolved main sequence stars and their ionization fronts in the ambient medium can also trigger further star formation. Therefore, in a complex environment with continuous star formation, it is often impossible to determine exactly the triggering mechanisms for star formation.

We have discussed the relationship between SNRs and the current and future star formation. In all cases, SNRs may alter the physical properties of the YSOs. For YSOs near SNRs, the passage of SNR shocks may strip the envelopes of YSOs and affect the accretion of mass. It would be interesting to examine the spectral energy distributions of these YSOs and search for differences from those of normal YSOs. YSOs near SNRs may intercept supernova ejecta so that heavy elements and radioactive material are incorporated into the circumstellar envelopes and disks. This is similar to what Looney et al. (2006) have suggested for our solar nebula. SNRs interacting 
with molecular clouds can inject heavy elements into the clouds for future star formation

It has often been assumed that an aged SNR both triggers the formation and enriches the circumstellar material of a YSO (e.g., Boss et al. 2010). However, our investigation of SNRs and star formation shows that SNRs with massive star progenitors are likely located near molecular clouds and their progenitors' or neighboring massive stars' ionization fronts and wind-blown bubbles can trigger star formation. It is thus common to have YSOs near an SNR to be enriched with the supernova ejecta, but their formation was not triggered by the SNR.

Finally, we note that clustered supernovae may produce large shell structures of sizes $\sim 100 \mathrm{pc}$, such as superbubbles blown by OB associations, or $\sim 1000$ pc, such as supergiant shells produced collectively by multi-generations of massive star formation (Chu 2008). These large shells have moderate expansion velocities, usually $\ll 50 \mathrm{~km} \mathrm{~s}^{-1}$, and thus may trigger star formation. Indeed, star formation has been observed around the rims of superbubbles, e.g., N44 (Chen et al. 2009),

and supergiant shells. e.g., LMC-4 (Yamaguchi et al. 2001; Book et al. 2009). While supernova explosions have led to the formation of these shell structures, the star formation is not attributed to any single SNR and therefore should not be referred to as "triggered by an SNR".

We thank the referee for his/her thorough reading and useful suggestions to improve the paper. We also thank Dr. Y. Fukui for providing the NANTEN data. This research was supported by NASA grants JPL 1290956 and JPL 1316421 and NSF grant AST 08-07323. JLP was supported by an appointment to the NASA Postdoctoral Program at the Jet Propulsion Laboratory, California Institute of Technology, administered by Oak Ridge Associated Universities through a contract with NASA.

\section{REFERENCES}

Banas, K. R., Hughes, J. P., Bronfman, L., \& Nyman, L.-A. 1997, ApJ, 480, 607

Bernasconi, P. A., \& Maeder, A. 1996, A\&A, 307, 829

Book, L. G., Chu, Y.-H., Gruendl, R. A., \& Fukui, Y. 2009, AJ, 137, 3599

Boss, A. P., Keiser, S. A., Ipatov, S. I., Myhill, E. A., \& Vanhala, H. A. T. 2010, ApJ, 708, 1268

Caulet, A., Gruendl, R. A., \& Chu, Y.-H. 2008, ApJ, 678, 200

Chen, C.-H. R., et al. 2009, ApJ, 695, 511

Chen, C.-H. R., et al. 2010, ApJ, submitted

Chen, Y., Wang, Q. D., Gotthelf, E. V., Jiang, B., Chu, Y.-H., \& Gruendl, R. 2006, ApJ, 651, 237

Chevalier, R. A., Kirshner, R. P., \& Raymond, J. C. 1980, ApJ, 235, 186 
Chu, Y.-H. 1997, AJ, 113, 1815

Chu, Y.-H. 2008, IAU Symposium, 250, 341

Chu, Y.-H., Dickel, J. R., Staveley-Smith, L., Osterberg, J., \& Smith, R. C. 1995, AJ, 109, 1729

Chu, Y.-H., \& Gruendl, R. A. 2008, Massive Star Formation: Observations Confront Theory, ASP Conf. Series, 387, 415

Chu, Y.-H., \& Kennicutt, R. C., Jr. 1988, AJ, 95, 1111

Chu, Y.-H., Kennicutt, R. C., Jr., Schommer, R. A., \& Laff, J. 1992, AJ, 103, 1545

Chu, Y.-H., Kennicutt, R. C., Snowden, S. L., Smith, R. C., Williams, R. M., \& Bomans, D. J. 1997, PASP, 109, 554

Chu, Y.-H., Mac Low, M.-M., García-Segura, G., Wakker, B., \& Kennicutt, R. C., Jr. 1993, ApJ, 414,213

Dunne, B. C., Points, S. D., \& Chu, Y.-H. 2001, ApJS, 136, 119

Fazio, G. G., et al. 2004, ApJS, 154, 10

Fukui, Y., Mizuno, N., Yamaguchi, R., Mizuno, A., \& Onishi, T. 2001, PASJ, 53, L41

Fukui, Y., et al. 2008, ApJS, 178, 56

Gouliermis, D. A., Chu, Y.-H., Henning, T., Brandner, W., Gruendl, R. A., Hennekemper, E., \& Hormuth, F. 2008, ApJ, 688, 1050

Gruendl, R. A., \& Chu, Y.-H. 2009, ApJS, 184, 172 (GC09)

Henize, K. G. 1956, ApJS, 2, 315

Hughes, A., et al. 2010, MNRAS, in press (astro-ph/1004.2094)

Hughes, J. P., et al. 1995, ApJ, 444, L81

Klimek, M., Points, S. D., \& Smith, C. 2006, BAAS, 38, 1113

Koo, B.-C., et al. 2008, ApJ, 673, L147

Looney, L. W., Tobin, J. J., \& Fields, B. D. 2006, ApJ, 652, 1755

Lucke, P. B., \& Hodge, P. W. 1970, AJ, 75, 171

Mathewson, D. S., Ford, V. L., Dopita, M. A., Tuohy, I. R., Long, K. S., \& Helfand, D. J. 1983, ApJS, 51, 345 
Mathewson, D. S., Ford, V. L., Dopita, M. A., Tuohy, I. R., Mills, B. Y., \& Turtle, A. J. 1984, ApJS, 55, 189

Mathewson, D. S., Ford, V. L., Tuohy, I. R., Mills, B. Y., Turtle, A. J., \& Helfand, D. J. 1985, ApJS, 58, 197

Matonick, D. M., \& Fesen, R. A. 1997, ApJS, 112, 49

Misselt, K. A., Clayton, G. C., \& Gordon, K. D. 1999, ApJ, 515, 128

Mostefaoui, S., Lugmair, G. W., \& Hoppe, P. 2005, ApJ, 625, 271

Nazé, Y., Chu, Y.-H., Points, S. D., Danforth, C. W., Rosado, M., \& Chen, C.-H. R. 2001, AJ, 122,921

Nicolet, B. 1978, A\&AS, 34, 1

Ott, J., et al. 2008, PASA, 25, 129

Points, S. D., Smith, R. C., Chu, Y.-H., Williams, R. N., \& Dickel, J. R. 2004, BAAS, 36, 1511

Rieke, G. H., et al. 2004, ApJS, 154, 25

Russell, S. C., \& Dopita, M. A. 1990, ApJS, 74, 93

Smith, R. C., Chu, Y.-H., Mac Low, M.-M., Oey, M. S., \& Klein, U. 1994, AJ, 108, 1266

Smith, R. C., \& The MCELS Team 1999, New Views of the Magellanic Clouds, IAUS, 190, 28

Tachibana, S., Huss, G. R., Kita, N. T., Shimoda, G., \& Morishita, Y. 2006, ApJ, 639, L87

van der Heyden, K. J., et al. 2002, A\&A, 392, 955

van der Marel, R. P., \& Cioni, M.-R. L. 2001, AJ, 122, 1807

Vanhala, H. A. T., \& Boss, A. P. 2002, ApJ, 575, 1144

Vanhala, H. A. T., \& Cameron, A. G. W. 1998, ApJ, 508, 291

Warren, J. S., Hughes, J. P., \& Slane, P. O. 2003, ApJ, 583, 260

Whitney, B. A., et al. 2008, AJ, 136, 18

Williams, R. M., Chu, Y.-H., Dickel, J. R., Smith, R. C., Milne, D. K., \& Winkler, P. F. 1999, ApJ, 514, 798

Williams, R. M., Petre, R., Chu, Y.-H., \& Chen, C.-H. R. 2000, ApJ, 536, L27

Yamaguchi, R., Mizuno, N., Onishi, T., Mizuno, A., \& Fukui, Y. 2001, PASJ, 53, 959 
Zaritsky, D., Harris, J., Thompson, I. B., \& Grebel, E. K. 2004, AJ, 128, 1606 

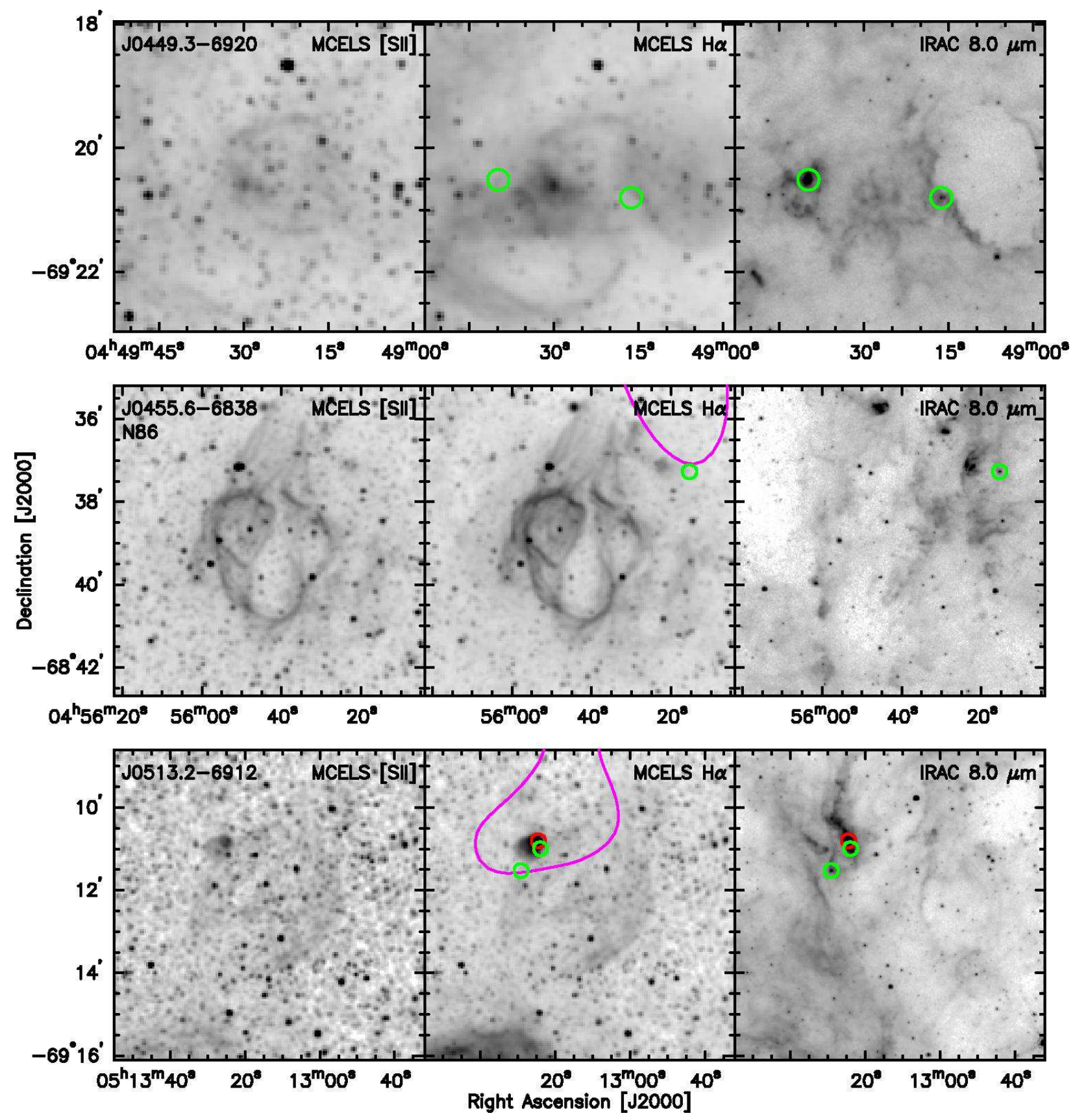

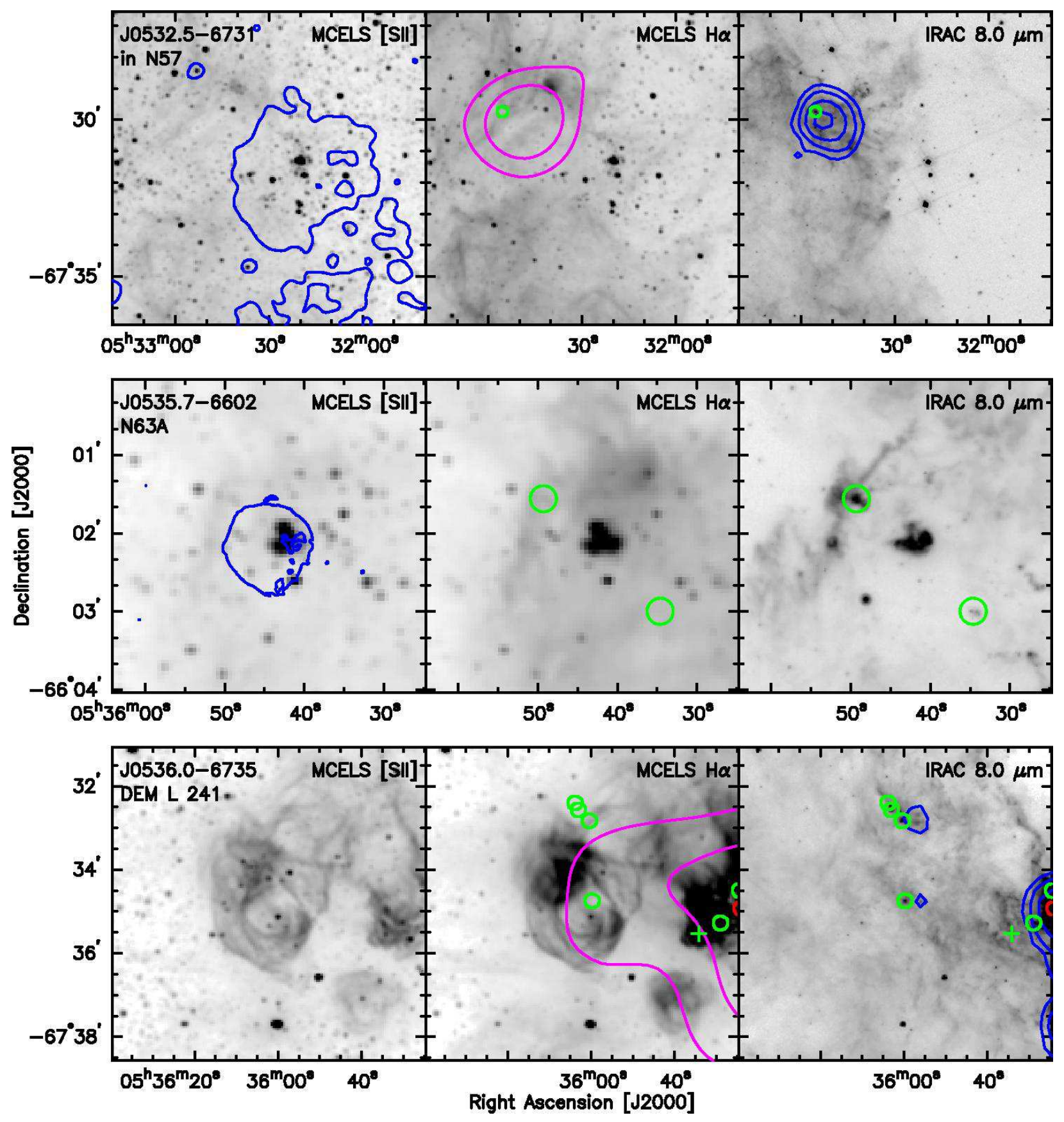

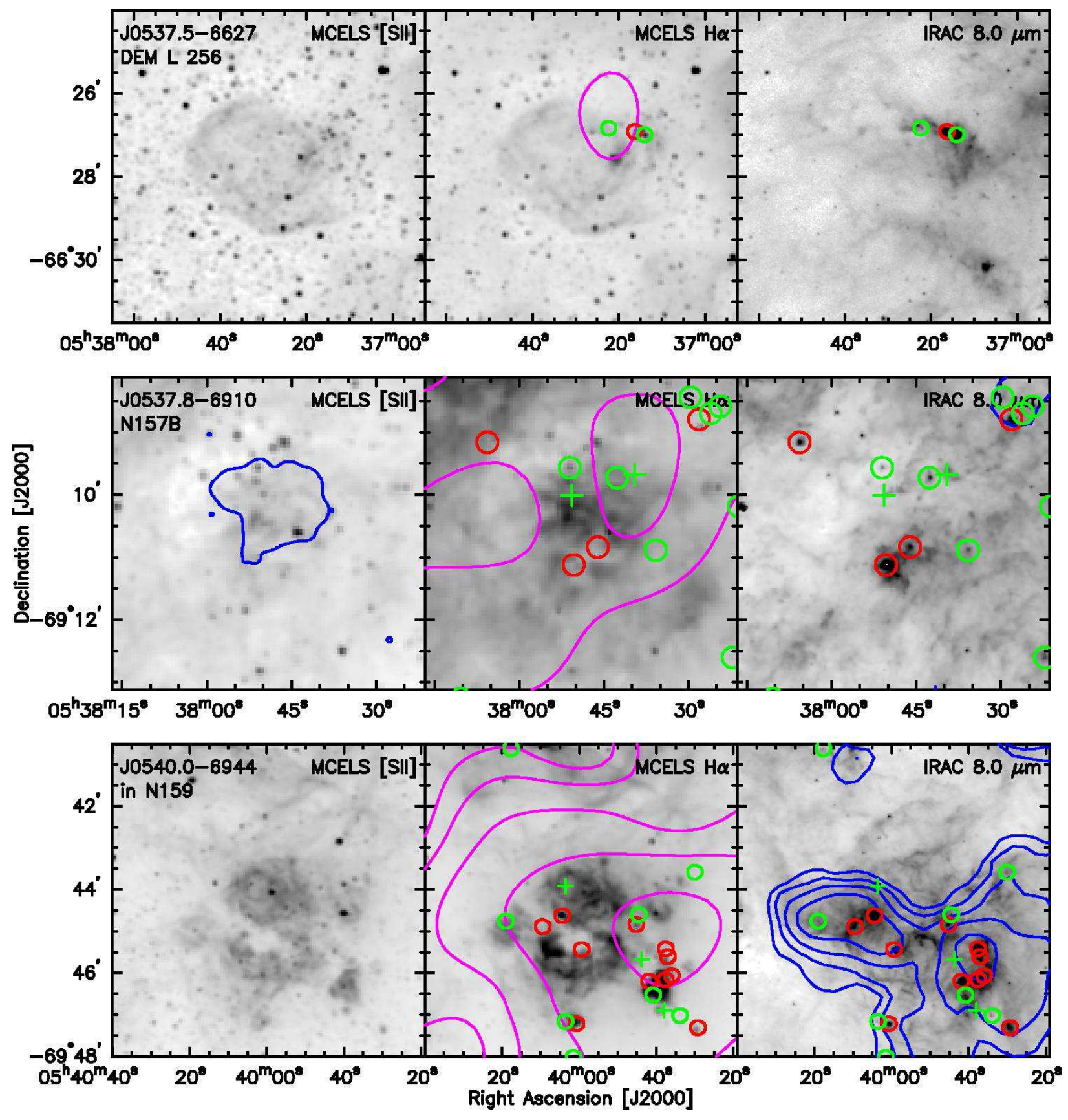


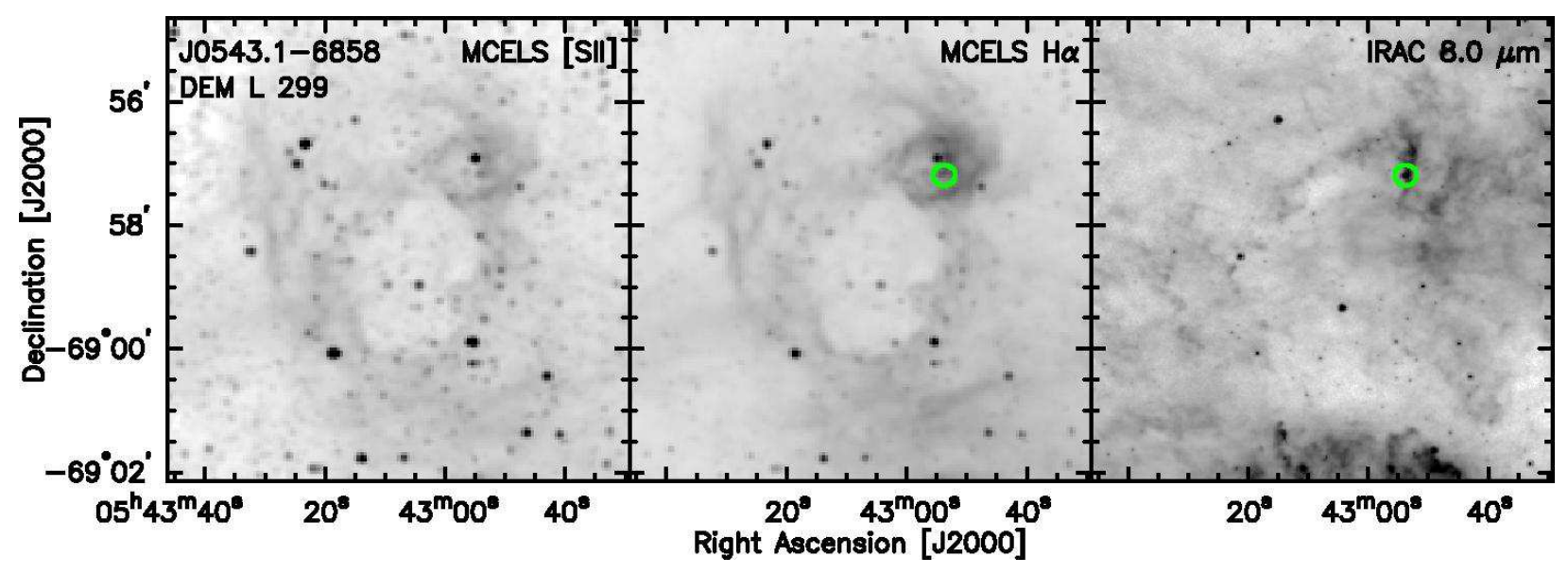

Fig. 1. - Images of SNRs that are associated with YSOs. For each remnant the three panels show images of MCELS [S II] (left), MCELS H $\alpha$ (middle), and Spitzer IRAC $8.0 \mu \mathrm{m}$ (right) emission. The emission line images are not continuum subtracted. Definite and probable YSOs from GC09 are marked with open circles while possible YSOs are indicated by a cross. Red symbols are used to mark the positions of massive YSOs while green symbols mark the intermediate-mass YSOs. Contours showing $\mathrm{CO}(\mathrm{J}=1 \rightarrow 0)$ emission from the NANTEN survey are overlaid on the $\mathrm{H} \alpha$ images while contours from the MAGMA survey are overlaid on the IRAC $8.0 \mu \mathrm{m}$ images. In both cases the contour levels used reflect the typical RMS noise level, $\sigma$, for the integrated intensity maps $\left(0.3 \mathrm{~K} \mathrm{~km} \mathrm{~s}^{-1}\right.$ and $0.5 \mathrm{~K} \mathrm{~km} \mathrm{~s}^{-1}$ for NANTEN and MAGMA, respectively) and follow a geometric progression starting at the $3 \sigma$ level (i.e., $3 \sigma, 6 \sigma, 12 \sigma, 24 \sigma, \ldots$ ). Note that MAGMA observations are available only for regions where GMCs are detected in the NANTEN survey. In three cases, J0532-6731, J0535.7-6602, and J0537.8-6910, we have overlaid a single X-ray contour on the [S II] image to show the extent of the remnant. 

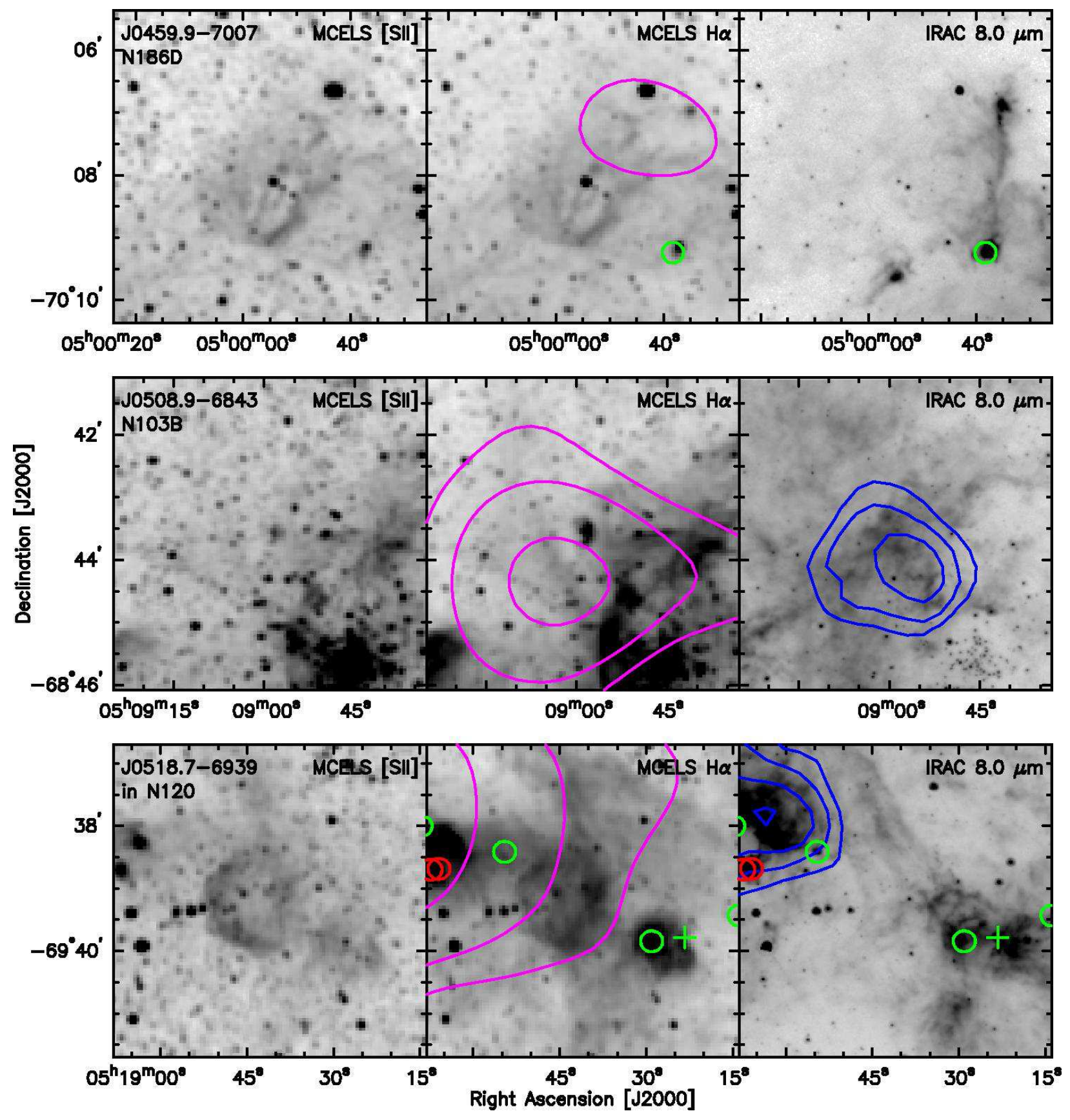

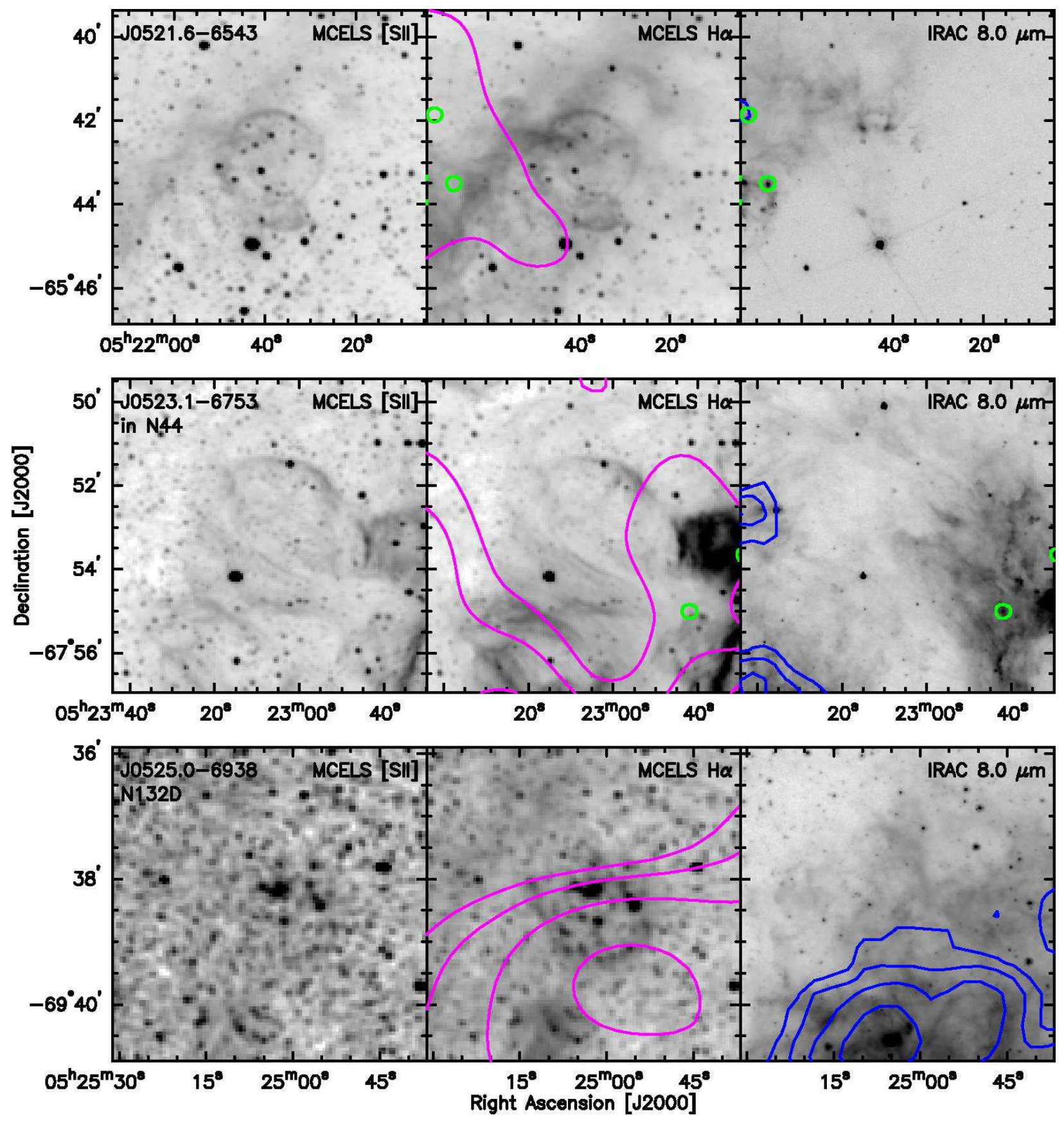

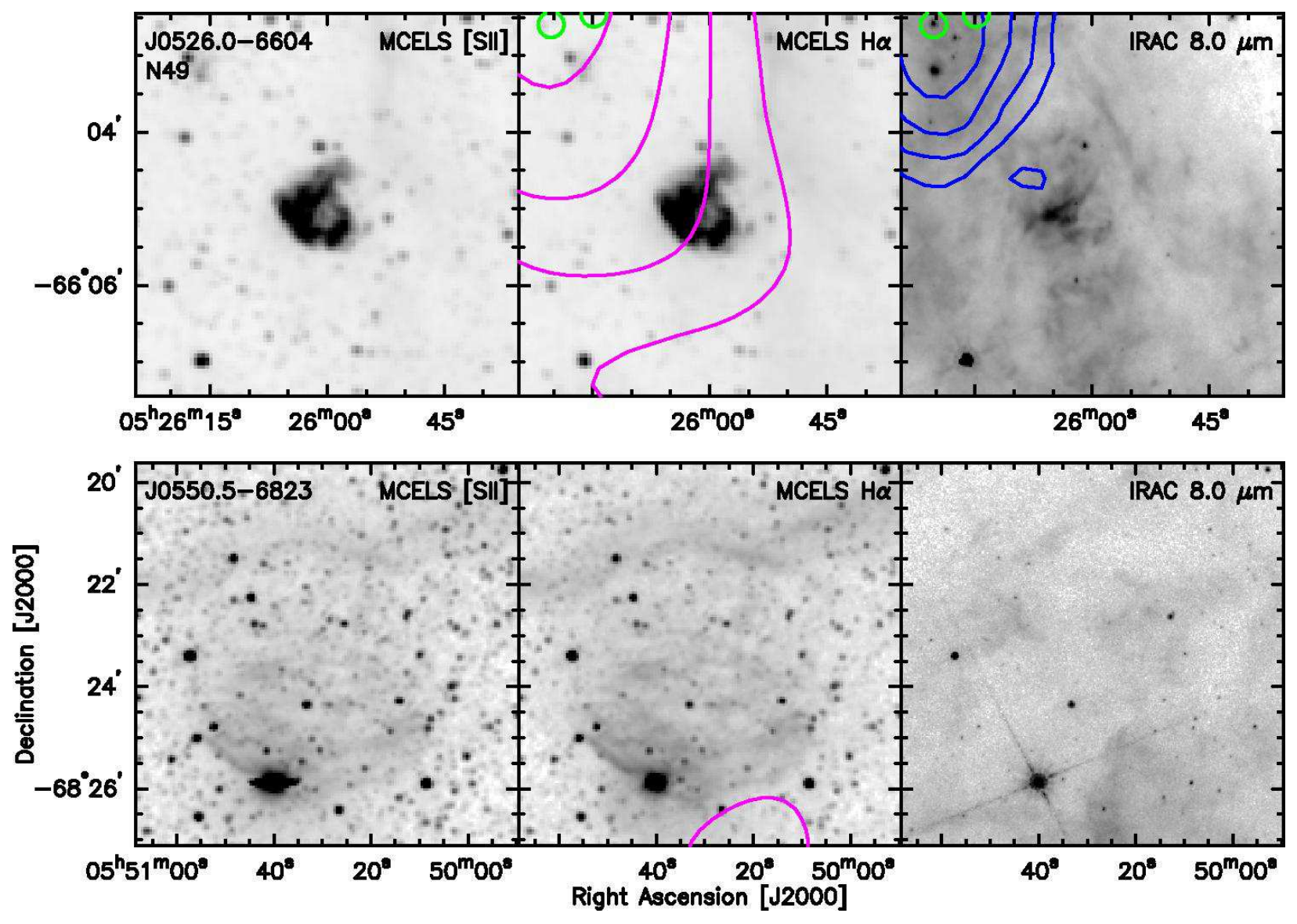

Fig. 2.- Images of SNRs that are associated with molecular clouds but no YSOs. See Figure 1 for a description of the contours. 


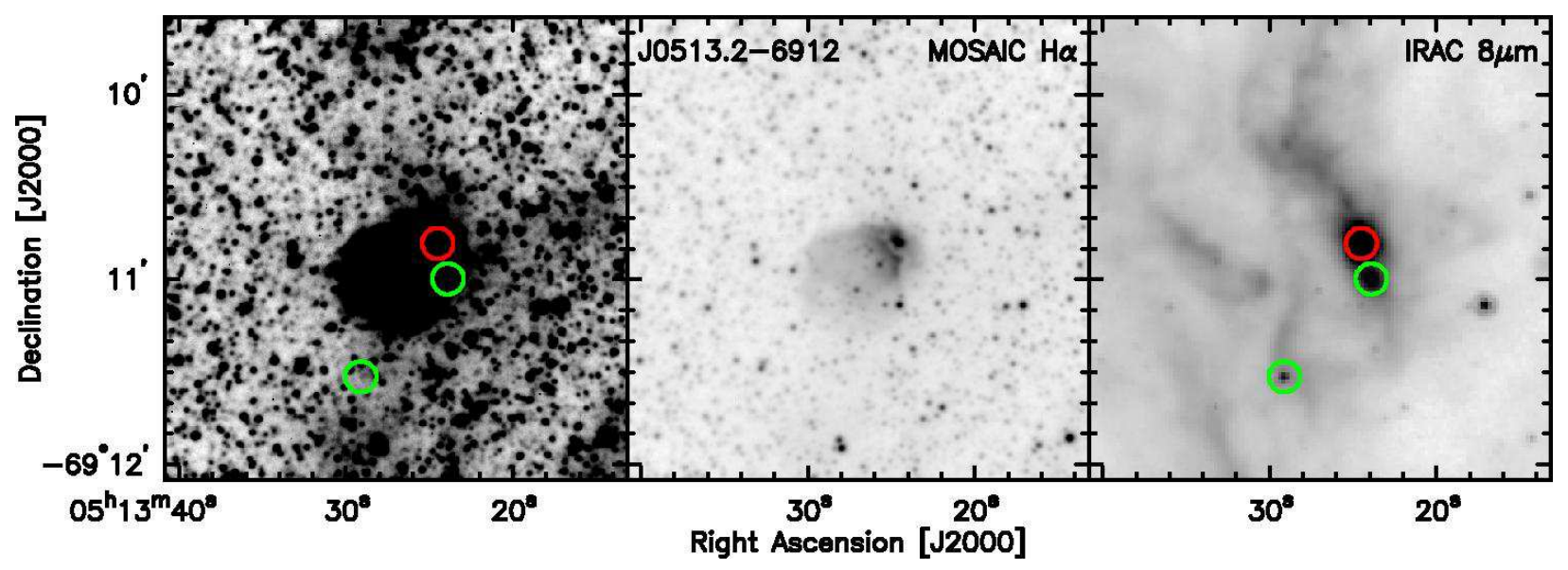

Fig. 3.- Close-up of the northeast rim of SNR J0513.2-6912 where the current YSOs and a small $\mathrm{H}$ II region are located. The left and central panels show an $\mathrm{H} \alpha$ image obtained with the MOSAIC camera on the CTIO 4m telescope. The left panel has a linear greyscale set to show the relation between the H II region and SNR shell rim, while central panel shows the details of the H II region structure. The right panel shows the $8 \mu \mathrm{m}$ emission. Locations of massive and intermediate-mass YSOs are marked with red and green open circles, respectively.

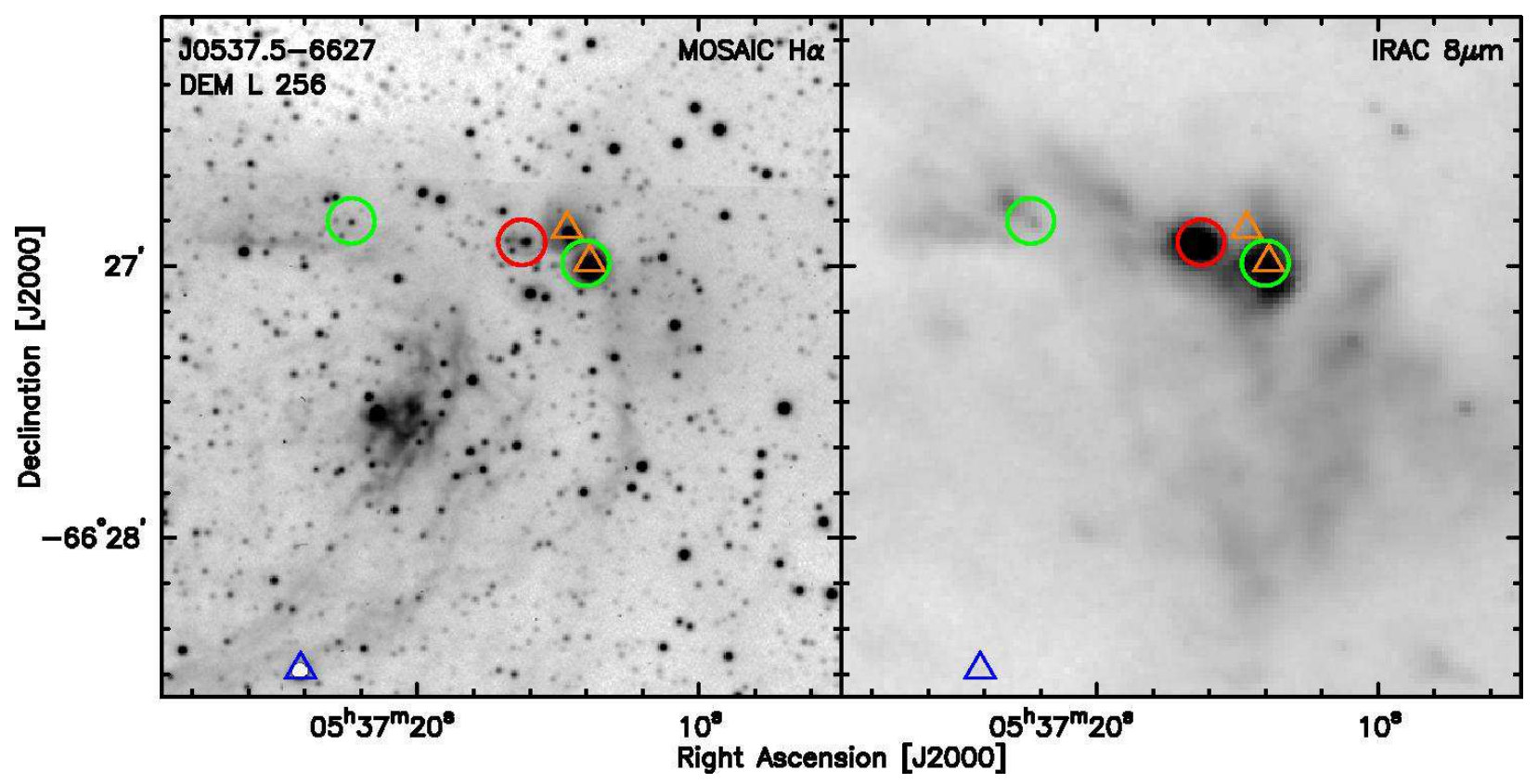

Fig. 4.- Close-up of the northwest rim of SNR J0537.5-6627, where the current YSOs are located. The left panel shows an $\mathrm{H} \alpha$ image acquired with the MOSAIC camera on the CTIO $4 \mathrm{~m}$ telescope, while the right panel shows the same region at $8 \mu \mathrm{m}$. The red and green open circles mark the locations of YSOs already shown in Figure 1. The locations of an O-type star and two early B-type stars are marked by blue and orange triangles, respectively. 


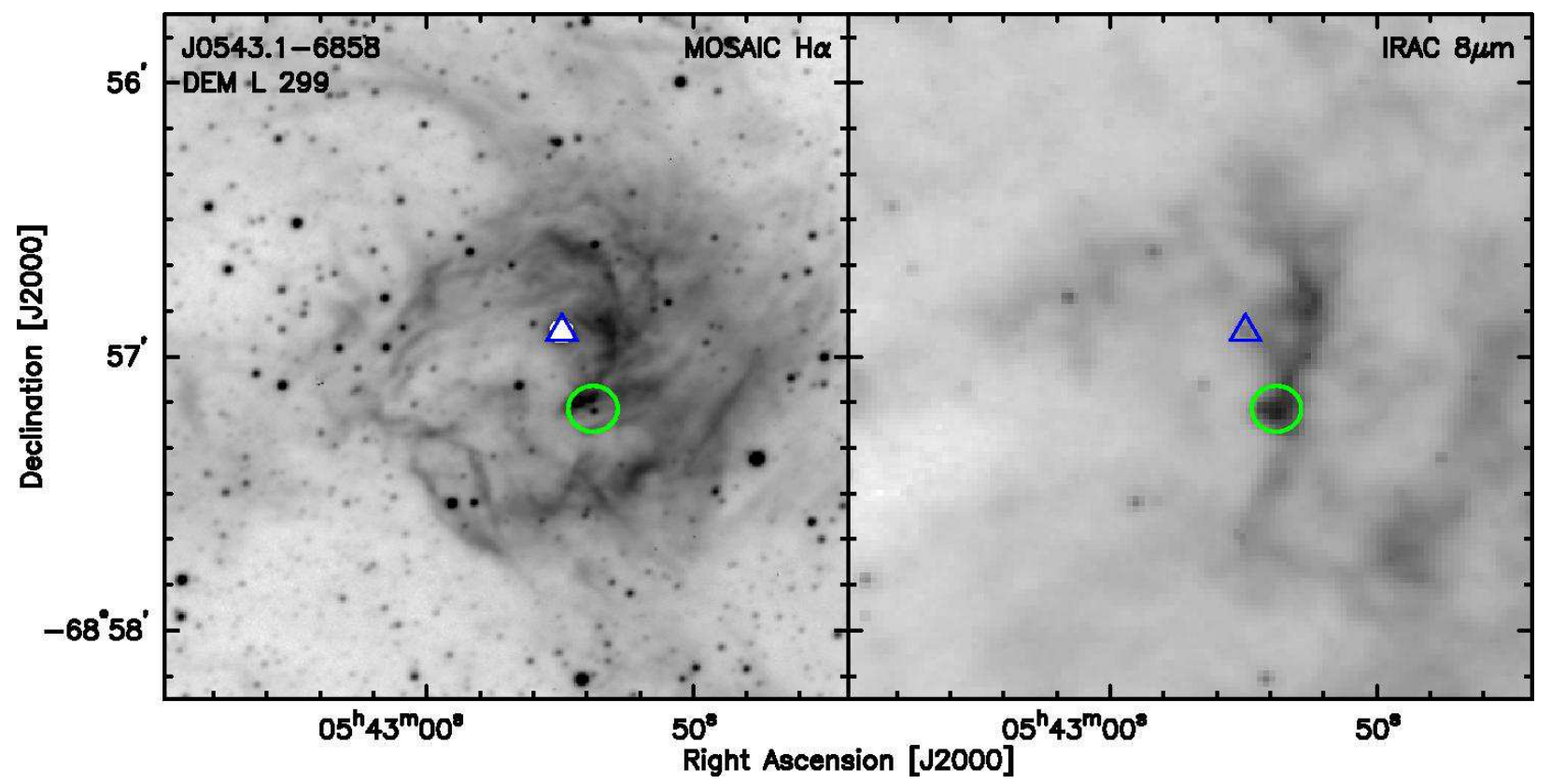

Fig. 5.- Close-up of the H II region on the northwest rim of SNR J0543.1-6858. The left panel shows an $\mathrm{H} \alpha$ image acquired with the MOSAIC camera on the CTIO $4 \mathrm{~m}$ telescope, while the right panel shows the same region at $8 \mu \mathrm{m}$. The open circle marks the location of the YSO, while the triangle marks the position of $\mathrm{Sk}-68155$ (saturated in the $\mathrm{H} \alpha$ image). 
Table 1. Supernova Remnants in the Large Magellanic Cloud

\begin{tabular}{|c|c|c|c|c|c|c|c|}
\hline $\begin{array}{l}\text { SNR } \\
\text { J2000 }\end{array}$ & $\begin{array}{l}\text { SNR } \\
\text { B1950 }\end{array}$ & $\begin{array}{l}\text { Other } \\
\text { Name }\end{array}$ & $\begin{array}{l}\text { RA (J2000) } \\
\text { (hh mm ss) }\end{array}$ & $\begin{array}{l}\text { Dec (J2000) } \\
\text { (ddd mm ss) }\end{array}$ & $\begin{array}{c}\text { Size } \\
(\operatorname{arcmin})\end{array}$ & YSOs & $\begin{array}{c}\text { NANTEN } \\
\text { CO }\end{array}$ \\
\hline $0448.4-6700$ & $0448-67.1$ & MCELS J0448-6659 & 044825 & -670012 & $4.5 \times 3.4$ & $\mathrm{~N}$ & $\mathrm{~N}$ \\
\hline $0449.3-6920$ & $0449-69.4$ & MCELS J0449-6921 & 044922 & -692025 & 2.0 & $\mathrm{Y}$ & $\mathrm{N}$ \\
\hline $0450.5-7050$ & $0450-70.9$ & SNR 0450-709 & 045030 & -705005 & $7.7 \times 5.3$ & $\mathrm{~N}$ & $\mathrm{~N}$ \\
\hline $0453.2-6655$ & $0453-66.9$ & SNR in N4 & 045314 & -665542 & 4.3 & $\mathrm{~N}$ & $\mathrm{~N}$ \\
\hline $0453.6-6829$ & $0453-68.5$ & SNR 0453-685 & 045338 & -682927 & 2.0 & $\mathrm{~N}$ & $\mathrm{~N}$ \\
\hline $0454.5-6713$ & $0454-67.2$ & SNR in N9 & 045433 & -671300 & $2.8 \times 2.2$ & $\mathrm{~N}$ & $\mathrm{~N}$ \\
\hline $0454.8-6625$ & $0454-66.5$ & N11L & 045450 & -662537 & $1.4 \times 1.0$ & $\mathrm{~N}$ & $\mathrm{~N}$ \\
\hline $0455.6-6838$ & $0455-68.7$ & N86 & 045544 & -683823 & $6.5 \times 3.5$ & $\mathrm{Y}$ & $\mathrm{Y}$ \\
\hline $0459.9-7007$ & $0500-70.2$ & N186D & 045956 & -700758 & $2.6 \times 2.3$ & $\mathrm{~N}$ & $\mathrm{Y}$ \\
\hline $0505.7-6752$ & $0505-67.9$ & DEM L71 & 050542 & -675239 & $1.5 \times 1.2$ & $\mathrm{~N}$ & $\mathrm{~N}$ \\
\hline $0505.9-6801$ & $0506-68.0$ & $\mathrm{~N} 23$ & 050555 & -680147 & $1.2 \times 0.8$ & $\mathrm{~N}$ & $\mathrm{~N}$ \\
\hline $0506.1-6541$ & $0506-65.8$ & DEM L72 & 050606 & -654108 & $6.4 \times 4.7$ & $\mathrm{~N}$ & $\mathrm{~N}$ \\
\hline $0508.9-6843$ & $0509-68.7$ & N103B & 050859 & -684335 & 0.50 & $\mathrm{~N}$ & $\mathrm{Y}$ \\
\hline $0509.5-6731$ & $0509-67.5$ & SNR 0509-675 & 050931 & -673117 & 0.56 & $\mathrm{~N}$ & $\mathrm{~N}$ \\
\hline $0513.2-6912$ & $0513-69.2$ & SNR 0513-692 & 051314 & -691208 & $4.5 \times 3.2$ & $\mathrm{Y}$ & $\mathrm{Y}$ \\
\hline $0518.7-6939$ & $0519-69.7$ & SNR in N120 & 051844 & -693909 & $1.6 \times 1.3$ & $\mathrm{~N}$ & $\mathrm{Y}$ \\
\hline $0519.5-6902$ & $0519-69.0$ & SNR 0519-690 & 051935 & -690209 & 0.55 & $\mathrm{~N}$ & $\mathrm{~N}$ \\
\hline $0519.7-6926$ & $0520-69.4$ & SNR 0520-694 & 051946 & -692600 & $2.4 \times 2.1$ & $\mathrm{~N}$ & $\mathrm{~N}$ \\
\hline $0521.6-6543$ & $0522-65.8$ & MCELS J0521-6542 & 052139 & -654310 & $3.0 \times 2.4$ & $\mathrm{~N}$ & $\mathrm{Y}$ \\
\hline $0523.1-6753$ & $0523-67.9$ & $\mathrm{SNR}$ in N44 & 052306 & -675306 & 3.5 & $\mathrm{~N}$ & $\mathrm{Y}$ \\
\hline $0524.3-6624$ & $0524-66.4$ & DEM L175a & 052418 & -662419 & $4.1 \times 2.8$ & $\mathrm{~N}$ & $\mathrm{~N}$ \\
\hline $0525.0-6938$ & $0525-69.6$ & $\mathrm{~N} 132 \mathrm{D}$ & 052504 & -693828 & $2.0 \times 1.5$ & $\mathrm{~N}$ & $\mathrm{Y}$ \\
\hline $0525.4-6559$ & $0525-66.0$ & N49B & 052525 & -655922 & $2.5 \times 2.3$ & $\mathrm{~N}$ & $\mathrm{~N}$ \\
\hline $0526.0-6604$ & $0525-66.1$ & N49 & 052600 & -660457 & $1.5 \times 1.3$ & $\mathrm{~N}$ & $\mathrm{Y}$ \\
\hline $0527.6-6912$ & $0528-69.2$ & SNR 0528-692 & 052739 & -691204 & $2.7 \times 2.0$ & $\mathrm{~N}$ & $\mathrm{~N}$ \\
\hline $0527.9-6550$ & $0527-65.8$ & DEM L204 & 052757 & -655000 & 4.5 & $\mathrm{~N}$ & $\mathrm{~N}$ \\
\hline $0530.7-7007$ & $0531-70.2$ & MCELS J0530-7008 & 053044 & -700710 & $3.5 \times 2.8$ & $\mathrm{~N}$ & $\mathrm{~N}$ \\
\hline $0531.9-7100$ & $0532-71.0$ & SNR in N206 & 053156 & -710019 & 3.0 & $\mathrm{~N}$ & $\mathrm{~N}$ \\
\hline $0532.5-6731$ & $0532-67.5$ & SNR $0532-675$ in N57 & 053230 & -673133 & 4.5 & $\mathrm{Y}$ & $\mathrm{Y}$ \\
\hline $0534.0-6955$ & $0534-69.9$ & SNR $0534-699$ & 053402 & -695505 & $1.7 \times 1.4$ & $\mathrm{~N}$ & $\mathrm{~N}$ \\
\hline $0534.3-7033$ & $0534-70.5$ & DEM L238 & 053418 & -703326 & $2.9 \times 2.5$ & $\mathrm{~N}$ & $\mathrm{~N}$ \\
\hline $0535.4-6916$ & $0535-69.3$ & SNR 1987A & 053528 & -691611 & $<0.1$ & $\mathrm{~N}$ & $\mathrm{~N}$ \\
\hline $0535.7-6602$ & $0535-66.0$ & $\mathrm{~N} 63 \mathrm{~A}$ & 053544 & -660214 & $1.4 \times 1.2$ & $\mathrm{Y}$ & $\mathrm{N}$ \\
\hline $0535.8-6918$ & $0536-69.3$ & Honeycomb & 053548 & -691804 & $1.4 \times 0.6$ & $\mathrm{~N}$ & $\mathrm{~N}$ \\
\hline $0536.0-6735$ & $0536-67.6$ & DEM L241 & 053603 & -673504 & 2.4 & $\mathrm{Y}$ & $\mathrm{Y}$ \\
\hline $0536.1-7038$ & $0536-70.6$ & DEM L249 & 053607 & -703837 & $3.0 \times 2.0$ & $\mathrm{~N}$ & $\mathrm{~N}$ \\
\hline $0537.5-6627$ & $0538-66.5$ & DEM L256 & 053730 & -662747 & $3.6 \times 2.8$ & Y & Y \\
\hline $0537.8-6910$ & $0538-69.1$ & N157B & 053748 & -691035 & $1.7 \times 1.2$ & $\mathrm{Y}$ & $\mathrm{Y}$ \\
\hline $0540.0-6944$ & $0540-69.7$ & SNR in N159 & 054000 & -694402 & 1.8 & $\mathrm{Y}$ & $\mathrm{Y}$ \\
\hline $0540.2-6919$ & $0540-69.3$ & N158A & 054012 & -691955 & $1.3 \times 1.1$ & $\mathrm{~N}$ & $\mathrm{~N}$ \\
\hline $0543.1-6858$ & $0543-68.9$ & DEM L299 & 054310 & -685849 & $5.8 \times 4.0$ & $\mathrm{Y}$ & $\mathrm{N}$ \\
\hline $0547.0-6942$ & $0547-69.7$ & DEM L316 B & 054700 & -694255 & $3.4 \times 2.8$ & $\mathrm{~N}$ & $\mathrm{~N}$ \\
\hline $0547.3-6941$ & $0547-69.7$ & DEM L316 A & 054722 & -694126 & 2.0 & $\mathrm{~N}$ & $\mathrm{~N}$ \\
\hline $0547.8-7024$ & $0548-70.4$ & SNR 0548-704 & 054749 & -702452 & $2.0 \times 1.8$ & $\mathrm{~N}$ & $\mathrm{~N}$ \\
\hline $0550.5-6823$ & $0551-68.4$ & SNR J0550-6823 & 055030 & -682322 & $5.2 \times 3.5$ & $\mathrm{~N}$ & $\mathrm{Y}$ \\
\hline
\end{tabular}


